\title{
Within-Person Variability in Urinary Bisphenol A Concentrations: Measurements from Specimens after Long-Term Frozen Storage
}

\author{
Pablo A. Nepomnaschy ${ }^{1,}{ }^{*}$, Donna Day Baird ${ }^{1, \S}$, Clarice R. Weinberg ${ }^{2}$, Jane A. Hoppin ${ }^{1}$, \\ Matthew P. Longnecker ${ }^{1}$, and Allen J. Wilcox ${ }^{1}$ \\ ${ }^{1}$ Epidemiology Branch, National Institute of Environmental Health Sciences. Department of Health \\ and Human Services, National Institutes of Health, Research Triangle Park, NC, USA \\ 2 Biostatistics Branch, National Institute of Environmental Health Sciences. Department of Health \\ and Human Services, National Institutes of Health, Research Triangle Park, NC, USA
}

\section{Abstract}

\begin{abstract}
Background-Bisphenol A (BPA) is an estrogenic contaminant of food and water associated with adverse developmental effects in laboratory animals. BPA has recently been linked to morbidity in adult humans, but studies of developmental effects in humans are methodologically more difficult. The ability to measure BPA in urine samples after long-term storage could aid in such studies. Because the half-life of BPA is $<6$ hours, a single measurement would be useful only if the environmental exposure is relatively constant over weeks or months. Our aim was to measure withinperson temporal variability in urinary BPA after 22-24 years of specimen storage, given that measurements suggested stability of BPA in the stored urine samples.
\end{abstract}

Methods-We measured total BPA concentration by mass spectrometry in first-morning urine samples from 60 premenopausal women. We selected from each woman's stored daily collections three urine samples approximately two and four weeks apart. Samples were selected from both the follicular and luteal phases of the menstrual cycle to assess cycle effects. Temporal variability was assessed with mixed model regression and correlations.

Results-BPA levels had an inter-quartile range from 1.1 to $3.1 \mathrm{ng} / \mathrm{mg}$ creatinine, slightly higher than levels in specimens from NHANES collected 3-11 years later. The Spearman correlation was approximately 0.5 for samples two weeks apart, and 0.3 for samples 4 weeks apart. Menstrual cycle phase did not influence levels. BPA tended to increase during the three-year collection period, but not significantly.

Conclusions-The similar distribution to NHANES samples and correlation of BPA levels taken at 2-week intervals provide indirect evidence that BPA is relatively stable during long-term freezer storage. The correlations indicate generally stable exposures over periods of weeks. These findings suggest that developmental effects of BPA exposure could be investigated with measurements from stored urine.

\section{Keywords}

bisphenol A; temporal variability; reproducibility; menstrual cycle 


\section{Introduction}

Bisphenol A (BPA; 2, 2-bis (4-hydroxyphenyl) propane) is a high volume, industrial chemical used in the manufacture of plastics and epoxy resins (Burridge, 2003). Leaching of BPA from polymer products, such as food-can liners and plastic bottles, leads to contamination of food and water, and direct exposure can occur from sources such as dental sealants (Kang et al., 2006). BPA has been found in urine, plasma, fetal plasma, placental tissue, follicular fluid, and breast milk (Vandenberg et al., 2007). By the time of the first US survey of BPA exposure (based on a subset of NHANES urine specimens from 1988-1994), exposure was nearly ubiquitous: $95 \%$ of those tested had detectable levels (Calafat et al., 2005).

A recent analysis of NHANES data found liver dysfunction and diabetes associated with BPA exposure in adult humans (Lang et al., 2008), but reproductive and developmental health concerns derive primarily from experimental studies with laboratory animals (Richter et al., 2007; vom Saal et al., 2007). The route of exposure appears important, with less toxicity from oral dosing than subcutaneous dosing (Wilhite et al., 2008). However, even at low levels, BPA has been reported to interfere with endogenous estrogens and disrupt normal estrogenic signaling. BPA disrupts thyroid hormone action, leads to meiotic aneuploidy, and adversely affects postnatal development (Welshons et al., 2006). Recent studies show higher risk of uterine and breast cancer following prenatal dosing (Newbold et al., 2007; Durando et al., 2007). Most studies of effects of BPA in humans have been limited by small numbers or other methodological problems including inadequate exposure assessment (Vandenberg et al., 2007).

Human studies of developmental effects, such as increased susceptibility to hormonally dependent cancers, would be facilitated by specimen collection during pregnancy, long-term storage, and BPA measurement later at the time when the developmental outcomes are assessed. However, chemical stability over long term storage has not been demonstrated. Urinary BPA-glucuronide breaks down quickly, freeing the BPA (Ye et al., 2007), but total BPA concentrations have been shown to remain relatively constant for at least a year of freezer storage (Califat, 2005). Longer-term storage effects have not been reported.

Another difficulty in exposure assessment in human studies is that BPA is rapidly metabolized. The estimated half-life is five to six hours (Volkel et al., 2002). Thus, exposure assessment based on single specimens would only reflect a person's chronic exposure if daily exposures are fairly constant. To our knowledge only three studies have examined changes over time in BPA exposure within individuals (Arakawa et al., 2004; Mahalingaiah et al, 2008; Teiltelbaum et al., 2008), and the largest was based on only 31 individuals with repeat values. All three studies assayed samples after short-term storage. Reproducibility in these studies was moderate suggesting that daily exposures are fairly constant over intervals of weeks to months.

We measured urinary BPA from 60 premenopausal women whose specimens had been in freezer storage for more than 20 years in order to evaluate by indirect assessments the longterm chemical stability of BPA. (BPA was not measured at time of urine collection, so direct comparison between measurements taken from the same specimen decades apart was not possible.) We examined the effect of year of urine collection on BPA concentrations and estimated the within-woman reproducibility over two and four week intervals. Additionally, we selected specimens from each woman during her follicular and luteal phases of the menstrual cycle in order to evaluate menstrual cycle effects on urinary BPA concentration. 


\section{Methods}

\section{Study Subjects and Urine Sample Selection}

Participants in the Early Pregnancy Study were 221 volunteers who enrolled at the time they discontinued birth control in order to become pregnant (Wilcox et al., 1988). Women agreed to collect daily first morning urine samples for up to six months during their attempt to conceive. Specimen collection took place from 1982 to 1986. Urine was collected in BPA-free, 30-ml wide-mouth polypropylene jars with screw tops. Samples were stored without preservatives in the participants' home freezers, with weekly pickup and transport to a central storage unit where they were kept at $-20^{\circ} \mathrm{C}$. Specimens were analyzed for reproductive hormones and then transferred to long-term storage vials (first in glass and later polypropylene) and again stored at $-20^{\circ} \mathrm{C}$. Thus, specimens had been thawed and refrozen at least twice before BPA measurement.

Sixty women were selected who had adequate quantities of urine from two ovulatory menstrual cycles. Similar to the total group of 221 participants (Wilcox et al. 1988), most of these 60 women were white $(94 \%)$ and their ages ranged from 21 to 42 years (mean $=29, \mathrm{SD}=4)$. Urine samples from these 60 women were collected in 1983 ( $n=77), 1984(n=46)$, and 1985 $(\mathrm{n}=57)$. Menstrual phase at time of sample collection was determined based on day of ovulation as estimated later from urinary estrogen and progesterone metabolite levels (Baird et al., 1991). For each woman, three samples were selected to include both follicular and luteal samples. The three samples are designated in chronological order as Time 1,2, and 3; in most cases Time 2 was during the luteal phase. For most women, the selected samples were two weeks apart. All samples had unique identifiers so that the laboratory could not identify specimens from the same woman. For 20 of the 180 collection days selected, we prepared two replicate samples as blind replicates. Thus, a total of 200 samples were analyzed. Specimens were shipped with dry ice by overnight freight to AXYS Laboratory (BC, Canada).

\section{Measurement of BPA and Creatinine}

The combination of free and conjugated BPA was measured. Deconjugation was performed with $\beta$-glucuronidase at $37^{\circ} \mathrm{C}$. A 4-methylumbelliferyl glucuronide solution was used for monitoring the deconjugation efficiency. Samples were extracted and cleaned using a Waters Oasis HLB solid phase extraction cartridge. The extract was then spiked with recovery standards. Analysis of sample extracts for bisphenol A was conducted using Waters 2690 or Waters 2795 HPLC coupled with a triple quadrapole mass spectrometer, running the manufacturer's MassLynx v.4.0 software. The mass spectrometer was run at unit mass resolution in the Multiple Reaction Monitoring mode. Based on spiked recovery standards, a "specimen detection limit" was determined for each sample by converting the area equivalents corresponding to 3 times the height of the chromatographic noise to a concentration (in the same way as peak areas are converted to concentrations). The "method detection limit" of the assay was calculated as the greater of either the concentration of the lowest calibration standard converted to a sample equivalent concentration, or the sample detection limit. This value was $0.18 \mathrm{ng} / \mathrm{ml}$.

Samples were analyzed in batches. The three specimens from a given woman were analyzed in the same batch for 58 of the 60 women. For the other two, one of their three specimens was analyzed in a separate batch. Each batch included a procedural blank, two spiked reference samples (one low and one high level concentration spike), and a reference sample in duplicate using lab stock urine for inter- and intra-batch comparisons. Intra- and inter-assay coefficients of variation (CV) were $14 \%$ and $17 \%$ respectively, based on these stock urine specimens. The intra-assay CV calculated based on our blind replicates was $28 \%$ which included one extremely non-concordant pair $(\mathrm{CV}$ for that duplicate pair $=109 \%)$. When the outlier was dropped, the 
$\mathrm{CV}$ was $22 \%$. No blind interassay CV was calculated because there were not enough replicates distributed among batches. Creatinine was assessed by the Jaffe assay (Taussky, 1954).

\section{Statistical Analyses}

We described the distribution of BPA values for the entire sample, for each of the three years of collection $(1983,1984,1985)$, and for each of the three sampling times for each individual (Time 1, Time 2, Time 3) using percentiles and geometric means. For analyses, specimens with BPA levels below the specimen-specific detection limit (SDL) were imputed by assigning a value equal to the SDL divided by the square root of 2 (Hornung and Reed, 1990). The initial descriptive analyses were conducted for both unadjusted and creatinine-adjusted BPA levels ( $\mathrm{ng} / \mathrm{ml}$ and $\mathrm{ng} / \mathrm{mg}$ creatinine, respectively), but further analyses used creatinine-adjusted concentrations. The distribution was right-skewed, so the natural logarithm of the measured BPA concentration was used in statistical analyses for which a normal distribution is optimal. Pearson and Spearman correlations were calculated between each of the three pair-wise comparisons (Times 1 and 2, Times 2 and 3, and Times 1 and 3). We examined the effect of sampling year and estimated within- and between-woman variation, as well as the effect of menstrual phase, using mixed model logistic regression with woman as a random effect. We estimated reliability based on all three measurements per woman using the intraclass correlation coefficient (ICC). ICCs and their 95\% confidence intervals (CIs) were calculated based on Shrout and Fleiss, 1979 by fitting a compound symmetry structure in SAS' mixed procedure using a "one random judge" method.

\section{Results}

BPA was detected in $91 \%$ of samples analyzed 22-24 years after collection. The $18(9 \%)$ specimens with non-detectable BPA levels were fairly evenly distributed among the 3 withinwoman sampling times ( 7 from Time 1, 5 from Time 2, and 6 from Time 3). Their SDLs ranged from $0.27 \mathrm{ng} / \mathrm{ml}$ to $1.7 \mathrm{ng} / \mathrm{ml}$ (mean of SDLs $=0.82 \mathrm{ng} / \mathrm{ml}$ ). None of the 60 women had undetectable levels in more than one of her three urine samples. Table 1 shows the distribution of creatinine adjusted and unadjusted BPA measurements. There was little reduction of variability with creatinine adjustment (SD of geometric mean was 2.6 for both) (Table 1). The overall geometric mean BPA value was $1.8 \mathrm{ng} / \mathrm{mg}$ of creatinine. The distributions of creatinineadjusted BPA concentration increased over the three sampling years (Table 1), consistent with a sharp rise in US BPA production during 1980-1985 (Chemical Economics Handbook, 2000). Creatinine-adjusted BPA distributions for the three within-participant sampling times (Time 1, Time 2, Time 3) were all very similar to the overall distribution (Table 1).

We found significant variability in urinary BPA concentrations both within-and betweenwomen, but within-woman variation was larger (within- woman variance $=0.53, \mathrm{SE}=0.07, \mathrm{p}$ $<.0001$; between-woman variance $=0.38, \mathrm{SE}=0.10, \mathrm{p}=0.0002$ ). The increase in BPA concentration during the three years of urine collection did not reach statistical significance in the mixed model $(\mathrm{p}=0.2)$. There was no evidence of an association with menstrual cycle phase $(\mathrm{p}=0.9)$.

Correlations between creatinine-adjusted BPA levels for the 60 women across sampling times are shown in Figure 1. The Spearman correlations between consecutive samples (Time 1-to-2 and Time 2 to-3) were both above 0.5 . The interval from Time 1 to Time 3 was approximately twice as long, with a correlation between those samples of 0.3 (Figure 1). To investigate whether specimens held in storage for the longest time would show lower correlations between samples, we examined Spearman correlations between samples from the same woman for the subset collected in 1983. The correlations were similar to those for the whole group (Time 1to- $2=0.68$, Time 2 -to- $3=0.47$, Time 1 -to- $3=0.45$ ). The ICC, based on data from all three time points for all 60 women was $0.43(95 \% \mathrm{CI}=0.31,0.56)$. 


\section{Discussion}

BPA was first synthesized more than a century ago, and its industrial use in the United States has grown exponentially over the last several decades (Burridge, 2003). Its estrogenic effects have been known since the 1930's (Tess, 1988). Urinary concentrations in the United States were first reported in 2005 based on a subset of the 1988-1994 NHANES III participants. The creatinine-adjusted geometric mean for the 210 women in the NHANES III cohort was $1.1 \mathrm{ng} /$ $\mathrm{mg}$ creatinine, with a $25^{\text {th }}$-to- $75^{\text {th }}$ percentile range of $0.7-3.0 \mathrm{ng} / \mathrm{mg}$ creatinine (Calafat et al., 2005). Our samples are from 3-11 years earlier (1983-1985) with a slightly higher mean (1.8 $\mathrm{ng} / \mathrm{mg}$ creatinine) and $25^{\text {th }}$-to- $75^{\text {th }}$ percentile range $(1.1-3.1 \mathrm{ng} / \mathrm{mg}$ creatinine). One potentially important source of BPA exposure, plastic liners in food cans, began before both data collection periods (Tess, 1988), so this exposure would affect both our sample and the more recent NHANES participants.

For our study BPA was measured by a commercial laboratory that adapted the methodology developed at the CDC (4). The CVs calculated from the laboratory reference urine sample were $14 \%$ and $17 \%$ for intra- and inter-assay CVs, although the intra-assay CV based on the blind replicate specimens was higher (28\%). We know of no published data on BPA CVs based on replicate specimens blinded to the laboratory. CVs based on blinded specimens can often be higher than those calculated from laboratory reference specimens because when the reference specimens have known concentrations, assays are re-run when measurements differ from their known values. This correction process cannot be followed with blind replicates.

Short-term experiments suggest that BPA is chemically stable in frozen urine for at least a year (Calafat et al., 2005). Long-term stability has not been directly demonstrated; this would require a comparison of measurements taken at time of sample collection and then again decades later. Our results indirectly support long-term stability. The mean urinary concentrations and the distribution of BPA levels in our sample are similar to (and in fact slightly higher than) those of the NHANES III samples from 3-11 years later (Calafat et al., 2005). The BPA concentrations tended to increase in our samples across the three years of urine collection consistent with the nearly $80 \%$ rise in US production during the early 80 s (Chemical Economics Handbook, 2007). Most convincing, however, are the correlations between samples from the same woman. Measurement showed Spearman correlations over 0.5 between samples taken at 2 -week intervals but a lower correlation (0.3) between samples taken 4 weeks apart. The relatively high short-term correlations, with a decline in correlation over time, would be unlikely if BPA in urine degraded in random ways over years of storage. Adding to the plausibility of long-term stability, the correlations we observed across 2-week intervals were similar to correlations across 2 -week intervals reported for recently-collected specimens (Teitelbaum et al., 2008). Finally, within-woman correlations were not lower for the subset of samples from 1983, the samples in storage the longest, again suggesting that longer time in storage was not related to increased degradation.

BPA that is ingested is absorbed nearly completely by the intestinal epithelium and rapidly conjugated. The half-life is less than six hours, and nearly all ingested BPA is excreted in urine within 96 hours (Volkel et al., 2002). Our data are from first-morning urine samples, so generally they reflect excretion of BPA ingested on the previous day, mostly the previous evening. First-morning urine samples from the same person might be expected to show more day-to-day concordance than spot urine specimens, because measurements will not be as affected by diurnal variability in ingestion. Our data show a correlation of over 0.5 for samples taken two weeks apart, which suggests that day-to-day exposures are similar in the short term. However, for samples taken four weeks apart, the correlation dropped to 0.3 , suggesting that exposure becomes more variable across longer time intervals. 
Three previous studies measured BPA from repeat samples from the same individual. One included 5 Japanese individuals (Arakawa et al., 2004), another 31 U.S. men and women (Mahalingaiah et al., 2008), and the third 29 New York City children (Teitelbaum et al., 2008). The latter is the only one of these to report correlations between samples taken at various time intervals, and they also found decreased correlations as time between samples increased (maximum interval was six months). Therefore, single measurements are unlikely to provide accurate estimates of long-term exposure to BPA. However, our data suggest that BPA is generally stable in freezer storage. This raises the possibility that developmental effects in humans might be investigated with specimens that have been collected at the appropriate gestational age and stored for later assay. The results provide support for further analyses to evaluate the effects of BPA on reproductive outcomes measured in the North Carolina Early Pregnancy Study.

\section{Acknowledgments}

This research was funded by the intramural program at the National Institute of Environmental Health Sciences, National Institutes of Health, HHS. The study was approved by the National Institute of Environmental Health Sciences Institutional Review Board, and participants gave informed consent.

The field manager of the North Carolina Early Pregnancy Study was Joy Pierce, and D. Robert McConnaughey manages the study data files. An earlier version of this manuscript was reviewed by Walter Rogan and Retha Newbold.

\section{References}

Arakawa C, Fujimaki K, Yoshinaga J, Imai H, Serizawa S, Shiraishi H. Daily urinary excretion of bisphenol A. Environ Health Prevent Med 2004;9:22-6.

Burridge E. Bisphenol A: product profile. Eur Chem News 2003 April;17:14-7.

Baird DD, Weinberg CR, Wilcox AJ, McConnaughey DR, Musey PI. Using the ratio of urinary estrogen and progesterone metabolites to estimate day of ovulation. Statist Med 1991;10:255-66.

Calafat AM, Kuklenyik Z, Reidy JA, Caudill SP, Ekong J, Needham LL. Urinary concentrations of bisphenol A and 4-nonylphenol in a human reference population. Environ Health Perspect 2005;113:391-5. [PubMed: 15811827]

Dodds EC, Lawson W. Synthetic oestrogenic agents without the phenanthrene nucleus. Nature 1936;137:996.

Durando M, Kass L, Piva J, Sonnenschein C, Soto AM, Munoz de Toro M. Prenatal bisphenol A exposure induces pre-neoplastic lesions in the mammary gland of Wistar rats. Environ health Perspect 2007;115:80-6. [PubMed: 17366824]

Greiner, EOC.; Kalin, T.; Nakamura, IK. Chemical Economics Handbook. SRI Consulting; Menlo Park, CA: 2007. CEH Product Review: Bisphenol A.

Hornung RW, Reed LD. Estimation of average concentration in the presence of nondetectable values. Appl Occup Environ Hyg 1990;5:46-51.

Kang JH, Kondo F, Katayama Y. Human exposure to bisphenol A. Toxicol 2006;226:79-89.

Lang IA, Galloway TS, Scarlett A, Henley WE, Depledge M, Wallace RB, Melzer D. Association of urinary bisphenol A concentration with medical disorders and laboratory abnormalities in adults. JAMA 2008;300:1303-1310. [PubMed: 18799442]

Mahalingaiah S, Meeker JD, Pearson KR, et al. Temporal variability and predictors of urinary bisphenal A concentrations in men and women. Environ Health Perspect 2008;116:173-8. [PubMed: 18288314]

Newbold RR, Jefferson WN, Padilla-Banks E. Long-term adverse effects of neonatal exposure to bisphenol A on the murine female reproductive tract. Reproductive Toxicology 2007;24:253-8. [PubMed: 17804194]

Richter CA, Birnbaum LS, Farabolllini F, et al. In vivo effects of bisphenol A in laboratory rodent studies. Reproductive Toxicology 2007;24:199-224. [PubMed: 17683900] 
Shrout P, Fleiss J. Intraclass correlations: uses in assessing rater reliability. Psychological Bulletin 1979;86:420-428. [PubMed: 18839484]

Taussky HH. A microcolorimetric determination of creatine in urine by the Jaffe reaction. J Biol Chem 1954;208:853-61. [PubMed: 13174594]

Teitelbaum SL, Britton JA, Calafat AM, et al. Temporal variability in urinary concentrations of phthalate metabolites, phytoestrogens and phenols among minority children in the United States. Environ Res 2008;107:257-269. [PubMed: 17976571]

Tess, RW. Epoxy resin coatings. In: May, CA., editor. Epoxy Resins: Chemistry and Technology. Vol. 2. New York: Marcel Dekker; 1988. p. 719-82.

Vandenberg LN, Hauser R, Marcus M, Olea N, Welshons WV. Human exposure to bisphenol A (BPA). Reproductive Toxicology 2007;24:139-77. [PubMed: 17825522]

Volkel W, Colnot T, Csanady GA, Filser JG, Dekant W. metabolism and kenetics of bisphenol A in humans at low doses following oral administration. Chem Res Toxicol 2002;15:1281-7. [PubMed: 12387626]

vom Saal FS, Akingbemi BT, Belcher SM, et al. Chapel Hill bisphenol A expert panel consensus statement: Integration of mechanisms, effects in animals and potential to impact human health at current levels of exposure. Reproductive Toxicology 2007;24:131-8. [PubMed: 17768031]

Welshons WV, Nagel SC, Vom Saal FS. Large effects from small exposures. III. Endocrine mechanisms mediating effects of bisphenol A at levels of human exposure. Endocrinology 2006;147(6 suppl):S56-69. [PubMed: 16690810]

Wilcox AJ, Weinberg CR, O'Connor JF, Baird DD, Schlatterer JP, Canfield RE, Armstrong EG, Nisula BC. Incidence of early loss of pregnancy. New Engl J Med 1988;319:189-94. [PubMed: 3393170]

Willhite CC, Ball GL, McLellan CJ. Derivation of a bispenol A oral reference dose (RfD) and drinkingwater equivalent concentration. J Toxicol Environ Health, Part B Critical Reviews 2008;11:69-146.

Ye X, Bishop AM, Reidy JA, Needham LL, Calafat AM. Temporal stability of the conjugated species of bisphenol A, parabens, and other environmental phenols in human urine. J Exp Sci Environ Epidemiol 2007;17:567-572. 


\section{Within-Woman Correlations}

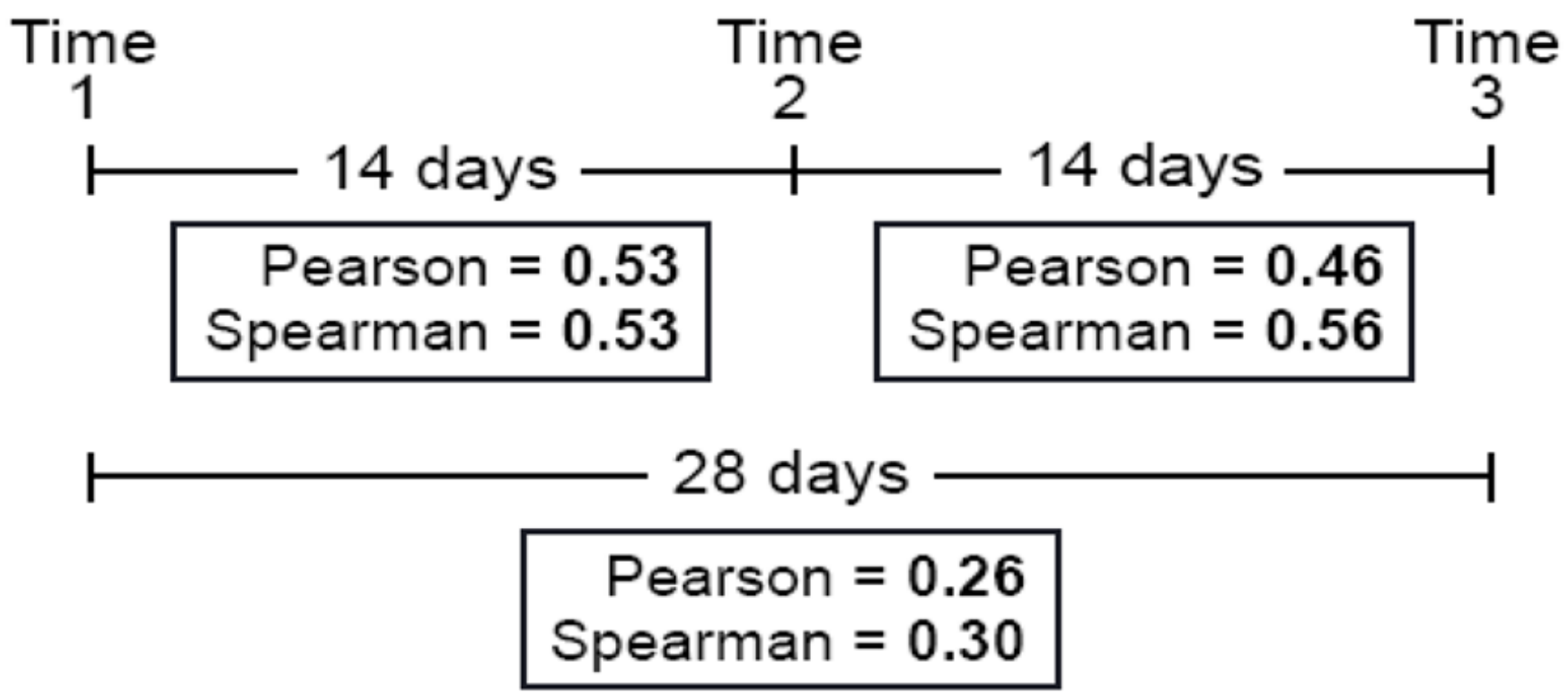

Figure 1.

Correlations between sample times for 60 women from the North Carolina Early Pregnancy Study. Data are based on creatinine-adjusted concentrations. Times shown between samples are medians (mean ${ }_{\mathrm{T} 1-\mathrm{T} 2}=16.6$ days, $\mathrm{SD}=6.8$; mean $\mathrm{T} 2-\mathrm{T} 3=15.3$ days, $\mathrm{SD}=8.5$; mean ${ }_{\mathrm{T} 1-\mathrm{T} 3}=31.8$ days, $\mathrm{SD}=11.2$ ). 
ब्ֶञ

임

엗ㄷำ

总

害 券

.

氙

ธั

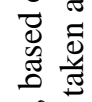

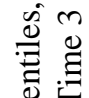

등

है

过

$\frac{0}{0}$

売节

$-3$

政

过

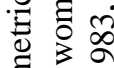

氕

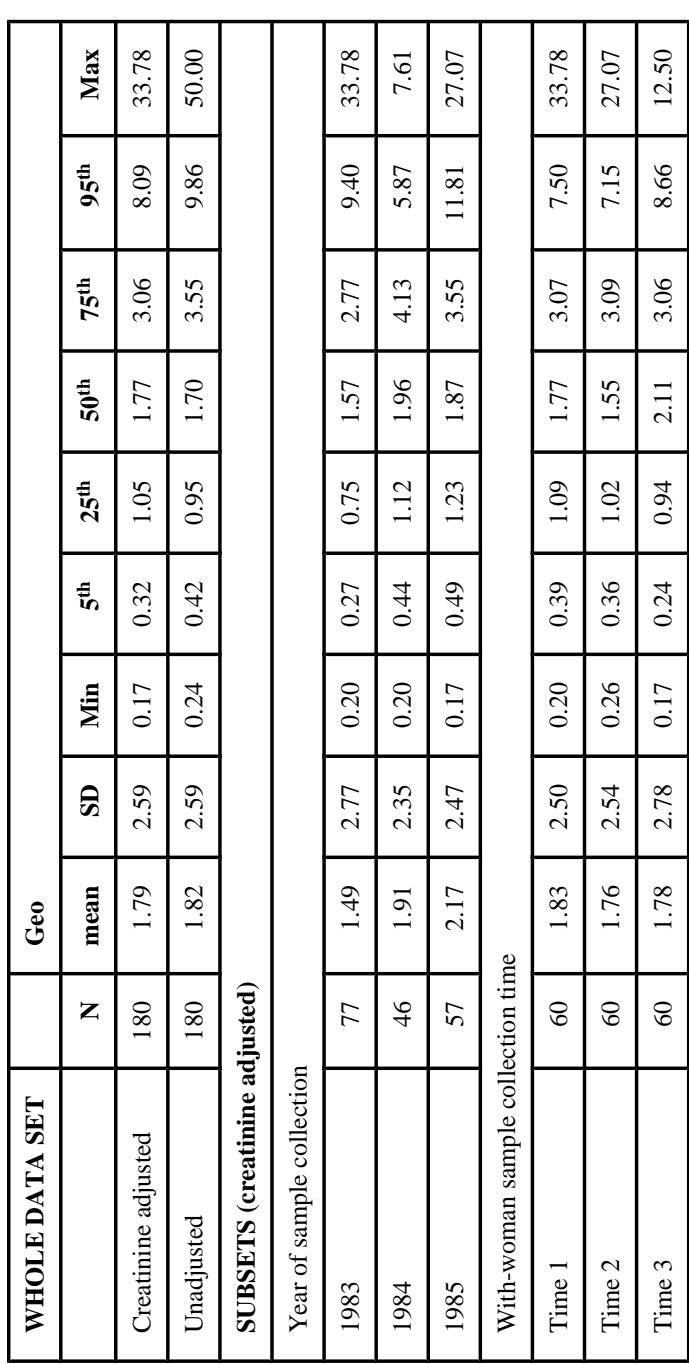

Environ Res. Author manuscript; available in PMC 2010 January 22. 